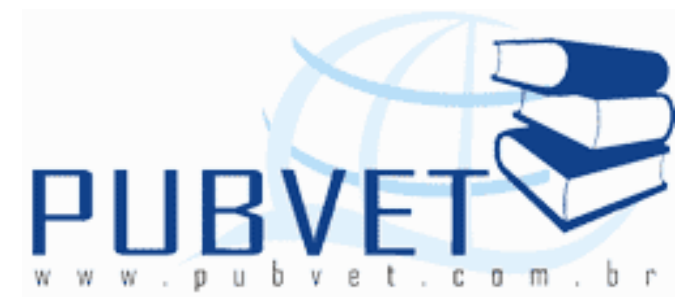

PUBVET, Publicações em Medicina Veterinária e Zootecnia.

Disponível em: <https://doi.org/10.31533/pubvet.v02n12a475.1-11>.

\title{
Hemangiossarcoma esplênico em gato: relato de caso
}

Leonardo Oliveira Trivilin ${ }^{1}$, Louisiane de Carvalho Nunes², Lenir Cardoso Porfírio ${ }^{2}$

${ }^{1}$ MV, Mestrando em Ciências Veterinárias - UFES

2 Professor Adjunto do Departamento de Medicina Veterinária - UFES

\section{RESUMO}

A neoplasia esplênica de origem no endotélio vascular é extremamente maligna e raramente é relatada em felinos. Objetivou-se com o presente artigo relatar um caso de hemangiossarcoma esplênico em felino demonstrando a característica da doença bem como as formas de diagnóstico utilizadas para a conclusão do caso. Um gato macho, 13 anos, foi levado ao HOVET-UFES com dificuldade respiratória grave e rapidamente morreu. À necropsia constatou-se, por meio de achados macro e microscópicos, o acometimento por hemangiossarcoma esplênico. Embora já tenha sido citado que o hemangiossarcoma esplênico em felinos é um neoplasma de ocorrência rara, o estabelecimento do diagnóstico precoce e correto poderia auxiliar na elaboração de tratamentos mais adequados.

PALAVRAS-CHAVE: baço, neoplasia, hemangiossarcoma, felino 


\title{
Splenic haemangiosarcoma in a cat: case report
}

\begin{abstract}
The splenic neoplasm of origin in the vascular endothelial is extremely malignant and rare it is told in cats. The report objective to tell a case of haemangiosarcoma splenic in feline demonstrating the characteristic of the illness as well the used forms of diagnosis for the conclusion of the case. A male cat, 13 years old, was taken to the HOVET-UFES with respiratory difficulty and quickly it came the death. To the necropsy of the animal if it evidenced, through macro and microscopical findings, the splen charge for haemangiosarcoma. Although already he has been cited that haemangiosarcoma in cats is a neoplasm of rare occurrence the establishment of the precocious diagnosis and correct it could assist more in the elaboration of adjusted treatments.
\end{abstract}

KEY WORDS: spleen, neoplasm, haemangiosarcoma, feline

\section{INTRODUÇÃO}

O hemangiossarcoma, hemangiotelioma ou angiossarcoma é uma neoplasia maligna, cuja origem é o endotélio vascular (McABEE et al., 2005; SMITH, 2003; NELSON e COUTO, 2001), possuindo uma incidência relatada de 0,3 a $2 \%$ ( McABEE et al., 2005).

Os locais de acometimento mais comumente relatados são o baço, átrio direito e o subcutâneo. No entanto, existem relatos de outros locais como cérebro, rim, intestinos, fígado, pulmões, bexiga, adrenais, próstata, entre outros (MERLO et al., 2002; NELSON e COUTO, 2001; SHARPE et al., 2000; HENRY et al., 1999; TUDOR e GREENLE, 1994; OKSANEN, 1978; ).

Biologicamente, o hemangiossarcoma assume um caráter altamente agressivo em relação à produção de metástases e infiltração, as quais ocorrem, na maioria das vezes, precocemente no decorrer da doença (McABEE et al., 2005; SMITH, 2003). 
Acomete animais idosos, sendo uma neoplasia comum em cães, mas raro em outras espécies (SMITH, 2003). Existem relatos de hemangiossarcoma em bovinos, eqüinos, suínos, caprinos, ovinos e humanos (JOHNS et al., 2005; PREZIUSO et al., 2002). Hemangiossarcoma esplênico em felinos não são comumente relatados, sendo a maior casuística relacionada ao acometimento cutâneo nessa espécie ${ }^{11}$. Relatos apontam que a idade média de gatos portadores dessa neoplasia varia de 10 a 13 anos (SCHULTHEISS, 2004; SHARPE et al., 2000; MILLER et al., 1992).

O diagnóstico de hemangiossarcoma pode ser obtido por citologia, por meio da citologia aspirativa com agulha fina (CAAF) ou por "Imprints". Já em derrames associados ao hemangiossarcoma ocorre uma dificuldade em se identificar o neoplasma. No entanto, em caso de diagnóstico presuntivo clínico ou citológico é fundamental a confirmação por exame histopatológico. Outra forma de diagnóstico é a ultra-sonografia, a qual se constitui em um método confiável para a avaliação em casos de hemangiossarcoma (NELSON e COUTO, 2001).

No que diz respeito ao tratamento as opções incluem cirurgias, quimioterapia e possivelmente radiação, porém, os tempos de sobrevida são invariavelmente curtos (usualmente menor que um ano), exceto para aqueles pacientes que apresentam hemangiossarcoma cutâneo. Além disso, outras opções para tratamento são correntemente investigados (SMITH, 2003). A quimioterapia se torna apropriada como adjuvante na cirurgia (MacEWEN, 2001). O uso de muramil tripeptídeo-fosfatidiletanolamina encapsulado em lipossomos (L-MTP-PE) após esplenectomia e quimioterapia combinada de doxorrubicina-ciclosfosfamida mostrou um incremento no intervalo de tempo livre de tumor, prolongando assim, o tempo de sobrevida (HAMMER, 2004).

Objetivou-se com 0 presente artigo relatar um caso de hemangiossarcoma esplênico em felino demonstrando a característica da doença bem como as formas de diagnóstico utilizadas para a conclusão do caso. 


\section{RELATO DO CASO}

Um gato macho, 13 anos, foi levado ao Hospital Veterinário (HOVET) do Centro de Ciências Agrárias da Universidade Federal do Espírito Santo após ser encontrado, pelo proprietário, com dificuldade respiratória e hipotermia.

O animal foi atendido no Setor de Clínica Médica de Pequenos Animais do HOVET apresentando sinais graves de dificuldade respiratória evoluindo rapidamente para um quadro de parada cardio-respiratória. Como conduta terapêutica de emergência fez-se ressuscitação cardio-respiratória, no entanto, o animal não resistiu e morreu.

O proprietário solicitou o exame necroscópico do animal. O procedimento foi realizado 30 minutos após a confirmação da morte, de acordo com o protocolo de necropsia estabelecido pelo Setor de Patologia Animal do HOVET.

Ao exame externo do cadáver observou-se obesidade e palidez das mucosas. À abertura da cavidade abdominal verificou-se a presença de hemoperitôneo, o qual foi mensurado num volume aproximado de $110 \mathrm{~mL}$. O exame do baço revelou uma massa avermelhada de aspecto irregular de aproximadamente $8,5 \mathrm{~cm}$, com área de ruptura (Figura 1).

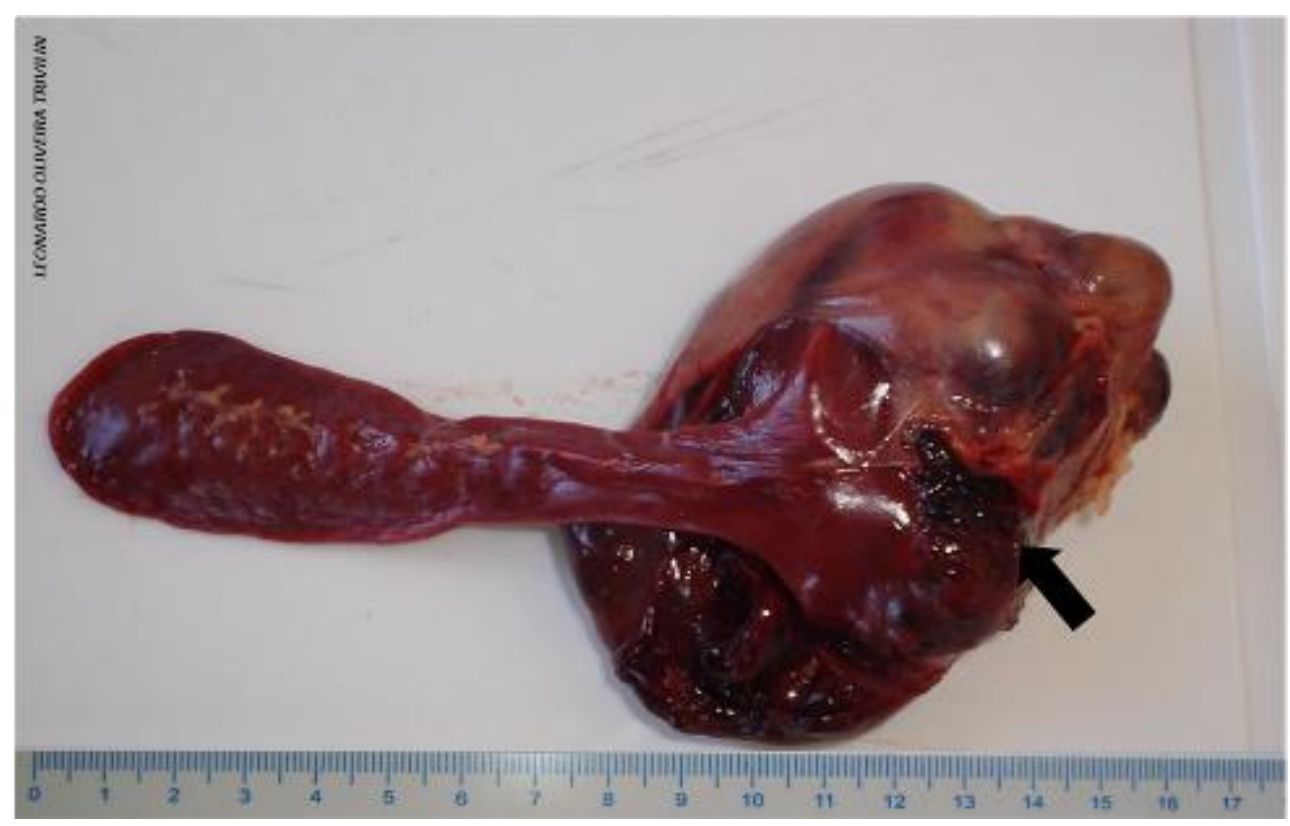

Figura 1 - Fotomacrografia do baço do felino doméstico deste relato, demonstrando massa neoplásica sugestiva de hemangiossarcoma com ruptura (seta). 
Antes da secção da massa neoplásica foi realizada citologia aspirativa por agulha fina (CAAF). O material corado pelo Giemsa revelou células de padrão ovalado, atípicas, com nucléolos centrais e evidentes que foram sugestivas de células mesenquimais de origem endotelial (Figura 2).

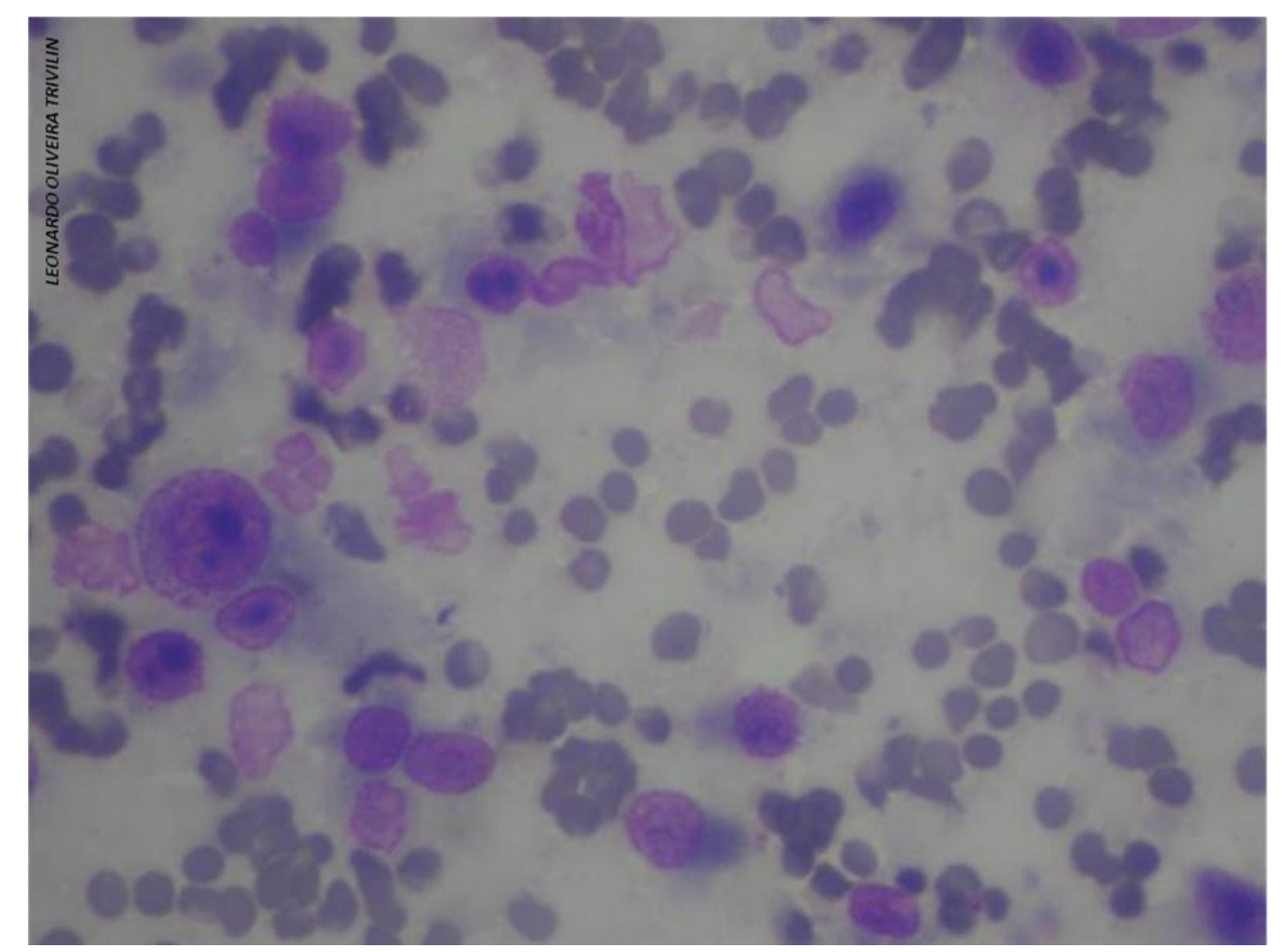

Figura 2 - Fotomicrografia do aspirado proveniente da massa neoplásica colhida através de CAAF. Giemsa, 400X.

Ao corte, a massa exibia cavitações com conteúdo sanguinolento. Ainda no baço havia a presença de um nódulo sugestivo de hiperplasia nodular senil de aproximadamente dois centímetros. O epíplon revelou uma pequena massa avermelhada de aproximadamente $2,5 \mathrm{~cm}$ de comprimento e o pâncreas uma massa avermelhada de aproximadamente um centímetro.

No tórax, o pulmão apresentava extensas áreas enfisematosas por todos os lobos. O coração apresentava uma discreta dilatação cardíaca direita além da presença de nodulações nas valvas mitral e tricúspide. No fígado foram encontradas pequenas massas semelhantes à encontrada no baço, sugerindo a 
ocorrência de metástase. Os órgãos do aparelho genito-urinário e cérebro não apresentaram alterações significantes.

Para a confirmação do diagnóstico foram colhidos fragmentos dos órgãos acometidos, fixados em solução de formalina tamponada a $10 \%$ e processados rotineiramente. Ao exame histopatológico observou-se no coração degeneração branda do músculo cardíaco, área focal de infiltrado inflamatório mononuclear e válvulas normais, e no baço e epíplon observou-se a presença de um neoplasma de alta celularidade com vários graus de atipia (pleomorfismo celular, anisocariose, células de núcleo arredondado, presença de nucléolos evidentes). Permeando as células neoplásicas foi observada uma neovascularização com presença de hemácias em seu interior. No fígado, além das alterações citadas, observou-se também congestão, hemorragia, proliferação dos ductos biliares, colestase, proliferação de tecido conjuntivo nos espaços porta, discreto infiltrado inflamatório mononuclear e degeneração de hepatócitos (Figura 3).

O pâncreas demonstrou a presença de hemangiossarcoma metastático além da presença de infiltrado inflamatório focal mononuclear, proliferação de tecido conjuntivo perivascular (esclerose vascular) e presença de células neoplásicas no interior dos vasos sangüíneos.

No presente relato, o animal apresentava-se com 13 anos quando da confirmação, por meio de necropsia, do acometimento por hemangiossarcoma esplênico, concordando com outros relatos para gatos domésticos pêlo curto (SHARPE et al., 2000). A média de idade de gatos acometidos com hemangiossarcoma é de nove anos variando entre cinco meses e 17 anos (MacEWEN, 2001).

Os achados de necropsia observados como hemoperitôneo foram descritos também em cães com hemangiossarcoma aos quais vieram a óbito após uma extensa hemorragia na cavidade peritoneal, pericárdio e cérebro (OKSANEN, 1978). Um estudo recente realizado em 30 cães com hemangiossarcoma revelou que 23 destes apresentaram hemoperitôneo após ruptura do tumor (MISCHKE et al., 2005). 


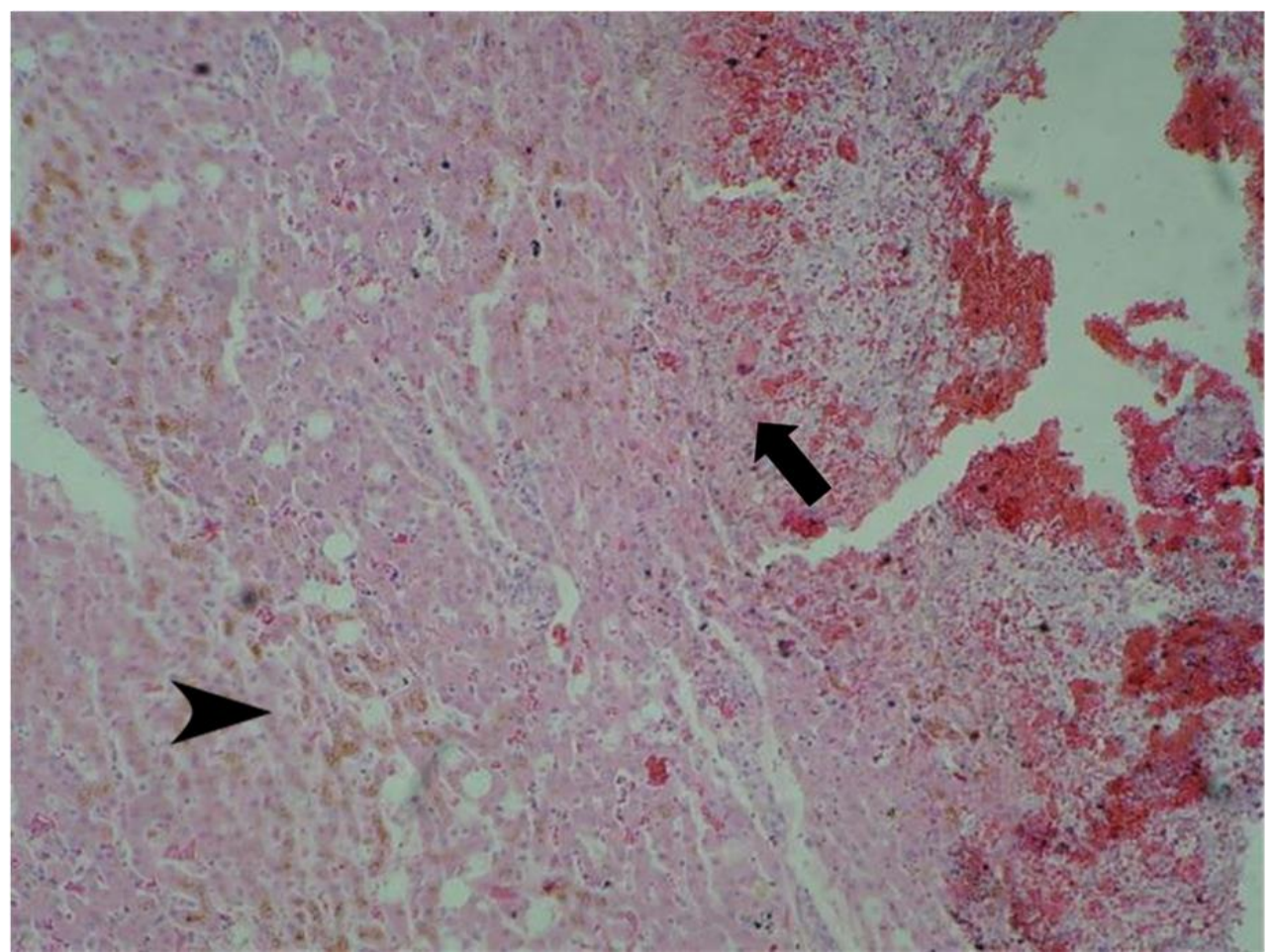

Figura 3 - Fotomicrografia de lâmina histológica do fígado do felino deste relato apresentando metástase de hemangiossarcoma (seta). Colestase também é observada nesta imagem (Cabeça da seta).H\&E, 100X.

De acordo com relatos, os hemangiossarcomas encontrados em cães necropsiados eram grandes, moles, cinzentos a vermelho escuro, nodulares, contendo hemorragias e áreas de necrose (OKSANEN, 1978). Ressalta-se ainda, a pobre circuncisão, o não encapsulamento e a freqüente aderência a órgãos adjacentes (MacEWEN, 2001). Esses aspectos também foram encontrados neste relato.

A presença de um nódulo no baço descrito neste relato corrobora os resultados obtidos por outros pesquisadores onde nódulos hiperplásicos, hematomas e a combinação desses eram $4 \%$ do total de casos de doenças esplênicas em gatos e num estudo de 455 casos de doenças esplênicas $37 \%$ eram neoplasias, dentre elas o hemangiossarcoma, com um comprometimento de omento e pâncreas em 3,7\% dos casos (17/455) (SPLANGER e CULBERTSON, 1992). 
Neste relato foram encontradas massas semelhantes àquela do baço no pâncreas, epíploon e fígado. Segundo relato, 50\% dos hemangiossarcomas podem ocorrer no fígado, baço ou mesentério, e que locais intra-abdominais com alta taxa metastática incluem o fígado, omento, diafragma, pâncreas e pulmão (MacEWEN, 2001). Outras regiões de metástase são peritônio, rins, miocárdio, tecido subcutâneo, cérebro (TUDOR e GREENLE, 1994), adrenais, bexiga, próstata, pleura e fêmur (OKSANEN, 1978).

Sobre o aspecto citológico, nos hemangiossarcomas freqüentemente notase baixa celularidade com numerosas células sanguíneas ao fundo da preparação. Espera-se encontrar eritrofagia aguda evidente ou hemorragia crônica e presença de hemossiderófagos. As células neoplásicas são pleomorfas, variando de grandes estruturas espiraladas a estreladas. 0 citoplasma apresenta-se basofílico com bordos celulares indistintos e vacuolização ocasional; alta proporção núcleo: citoplasma, núcleos ovais com cromatina grosseira e múltiplos nucléolos proeminentes (RASKIN e MEYER, 2003).

Em um estudo, o uso da CAAF revelou uma população de células pleomórficas com bordo celular mal definida, núcleos ovais e hipercromáticos e membranas nucleares irregulares (DELACRUZ et al., 2005). Achados semelhantes foram observados neste estudo, tornando-se a CAAF importante para o processo de conclusão do diagnóstico.

Uma das razões da CAAF não ser utilizada como método de diagnóstico em casos de neoplasias esplênicas deve-se ao fato de que o procedimento pode resultar em complicações significantes. Com isso, recentemente estudos têm demonstrado que a CAAF é tão segura quanto a biopsia devido ao pequeno tamanho da agulha usada, mesmo sendo o baço um órgão com elevada vascularização. Em geral a CAAF promove uma melhor amostra que a biopsia isso porque ela promove um acesso multidirecional e o grande número de passagens (DELACRUZ et al., 2005).

Histologicamente, as células neoplásicas são altamente pleomórfas, enfileirando-se em forma de eixo a poligonal ou ovóide formando, usualmente, 
uma fenda vascular reconhecível ou canais em alguma parte do tumor. As células que revestem essa fendas possuem núcleo proeminente hipercromático e pleomórfico. Figuras de mitose são freqüentes e em algumas áreas o estroma entre as fendas é acelular, hialino e claramente eosinofílico. Pode haver grandes áreas de hemorragia com poucas células imitando hematoma (GOLDSCHMIDT e HENDRICK, 2002).

A maioria dos hemangiossarcomas compreende células endoteliais pleomórficas com tamanho variado, e enfileiradas com espaços vasculares preenchidos com sangue (MCABEE et al., 2005). Outros autores acrescentam ainda a caracterização dos hemangiossarcomas por proliferação neoplásica com áreas focais ou difusas de componentes vasoformadores mostrando canais cavernosos e arborizantes (VALBUENA et al., 2005).

Para o fechamento do diagnóstico neste relato foi imprescindível a utilização do exame histopatológico, tanto da massa principal, quanto dos outros presentes nos órgãos como o fígado, pâncreas e epíplon. Os achados foram compatíveis com aqueles de outros autores, o que favoreceu 0 diagnóstico. A CAAF mostrou-se de grande importância neste caso, proporcionando um direcionamento para o fechamento do caso e liberação de um diagnóstico preciso.

\section{CONSIDERAÇÕES FINAIS}

Embora já tenha sido citado que o hemangiossarcoma esplênico em felinos é uma neoplasia de ocorrência rara o estabelecimento do diagnóstico precoce e correto poderia auxiliar na elaboração de tratamentos mais adequados.

Nesse relato o animal foi levado ao HOVET em estado grave evoluindo para morte em pouco tempo, impossibilitando a utilização de outros recursos diagnósticos, como a ultra-sonografia, por exemplo, ou mesmo a análise laboratorial do líquido cavitário abdominal (caracterizado post mortem como hemoperitôneo) para dignosticar definitivamente o quadro clínico do animal. 
Pelo fato do animal apresentar, clinicamente, quadro respiratório grave, outras suspeitas diagnósticas poderiam ser levantadas, no entanto, tratava-se de um caso de ruptura de massa neoplásica esplênica e choque hipovolêmico.

A utilização de métodos diagnósticos como ultra-sonografia, citologia guiada por ultra-som ou mesmo laparotomia exploratória seriam alternativas valiosas para o estabelecimento do diagnóstico e direcionamento para o tratamento adequado.

A importância do relato de hemangiossarcoma esplênico em felinos está na descrição, neste caso, da apresentação desta doença, bem como sua evolução, favorecendo aos profissionais lançar mão de diversas formas diagnósticas para a conclusão de casos semelhantes.

\section{REFERÊNCIAS}

SMITH, A. N. Hemangiosarcoma in dogs and cats. The Veterinary Clinics of North America. Small animal practice, n 33, p. 533-52, 2003

MCABEE, K. P.; LUDWIG, L. L.; BERGMAN, P. J. and NEWMAN, S. J. Feline cutaneous hemangiosarcoma: a retrospective study of 18 cases (1998-2003). Journal of the Americam Animal Hospital Association, n 41, p. 110-116, 2005

NELSON, R. W. e COUTO, C. G. Medicina Interna de Pequenos Animais, 2 ed. Rio de Janeiro: Guanabara Koogan, p. 897-898, 2001

TUDOR, K. e GREENLE, P. Cerebral metastatic hemangiosarcoma in the cat. Feline Practice, $\mathrm{n}$ 22 , p. $20-21,1994$

HENRY, C. J.; TURNQUIST, S. E.; SMITH, A.; GRAHAM, J. C.; THAMM, D. H.; O'BRIEN, M. and CLIFFORD, C. A. Primary renal tumours in cats: 19 cases (1992-1998). Journal of Feline Medicine \& Surgery, n 3, v 1, p. 165-170, 1999

MERLO, M.; BO, S. e RATTO, A. Primary right atrium haemangiosarcoma in a cat. Journal of Feline Medicine \& Surgery, n 1, v 4, p. 61-64, 2002

OKSANEN, A. Haemangiosarcoma in dogs. Journal of Comparative Pathology, n 4, v 88, p. 585-595, 1978

SHARPE, A.; CANNON, M. J.; LUCKE, V. M. e DAY, M. J. Intestinal haemangiosarcoma in the cat: clinical and pathological features of four cases. The Journal of Samll Animal Practice, n 9, v 41, p. 411-415, 2000 
Trivilin, L.O., Nunes, L.C. e Porfirio, L.C. Hemangiossarcoma esplênico em gato: relato de caso. PUBVET, Londrina, V. 2, N. 50, Art\#475, Dez3, 2008.

PREZIUSO, S.; TACCINI, E.; ROSSI, G.; BRACA, G. e RENZONI, G. Cutaneous haemangiosarcoma in a sheep: morphological, histopathological and immunohistochemical observations. Journal of Comparative Pathology, n 1, v 127, p. 72-75, 2002

JOHNS, I.; STEPHEN, J. O.; DEL PIERO, F.; RICHARDSON, D. W. e WILKINS, P. A. Hemangiosarcoma in 11 young horses. Journal of Veterinary Internal Medicine, $n$ 19, $p$. 564-570, 2005

SCHULTHEISS, P. C. A retrospective study of visceral and nonvisceral hemangiosarcoma and hemangiomas in domestic animals. Journal of Veterinary Diagnostic Investigation, $\mathrm{n} 16$, p. $522-526,2004$

MILLER, M. A.; RAMOS, J. A. e KREEGER, J. M. Cutaneous vascular neoplasia in 15 cats: clinical, morphological and immunohistochemical studies. Veterinary Pathology, n 29, p. 329-336, 1992

MacEWEN, E. G. Miscellaneous Tumors. In: Small Animal Clinical Oncology. 3 ed., Philadelphia: WB Saunders, cap. 29, p. 639-646, 2001

HAMMER, A. Hemangiossarcoma. In: Segredos em oncologia veterinária respostas necessárias ao dia-a-dia em rounds, na clínica, em exames orais e escritos. Porto Alegre: Artmed, cap. 33, p. 253-260, 2004

MISCHKE, R.; WOHLSEIN, P. e SCHOON, H. A. Detection of fibrin generation alterations in dogs with haemangiosarcoma using resonance thrombography. Thrombosis Research, $n$, $v$ 115 , p. $229-238,2005$

RASKIN, R. E. e MEYER, D. J. Atlas de Citologia de Cães e Gatos. São Paulo: Roca, p. 64, 2003

DELACRUZ, V.; JORDA, M.; FERNANDES, C. G.; BENEDETTO, P. e GANJEI, P. Fine-needle aspiration diagnosis of angiosarcoma of the spleen: a case report and review of the literature. Archives of Pathology and Laboratory Medicine, n 8, v 129, p. 1054-1056, 2005

SPLANGER, W. L. e CULBERTSON, M. R. Prevalence and type of splenic diseases in cats: 455 cases (1985-1991). Journal of the American Veterinary Medical Association, n 201, p. 773-776, 1992

GOLDSCHMIDT, M. H. e HENDRICK, M. J. Tumors of the skin and soft tissues. In: Tumors in Domestic Animals, 4 ed. Iowa: Iowa State Press, cap. 2, p. 99-100, 2002

VALBUENA, J. R.; LEVENBACK, C.; MANSFIELD, P.; LIU, J. Angiosarcoma of the spleen clinically presenting as metastatic ovarian cancer. A case report and review of the literature. Annals of diagnostic pathology, n 5, v 9, p. 289-92, 2005 